Int. J. Dev. Biol. 54: 1323-1328 (2010)

doi: $10.1387 /$ ijdb.092973jp

\title{
Active loss of DNA methylation in two-cell stage goat embryos
}

\author{
JUNG S. PARK1 ${ }^{1}$, DOOSOO LEE ${ }^{2}$, SUNWHA CHO ${ }^{1}$, SANG-TAE SHIN*,2 and YONG-KOOK KANG ${ }^{*, 1}$ \\ ${ }^{1}$ Development and Differentiation Research Center, KRIBB, Daejon and \\ ${ }^{2}$ College of Veterinary Medicine, Chungnam National University, Daejon, South Korea
}

\begin{abstract}
Early mammalian embryos are thought to gain nuclear totipotency through DNA methylation reprogramming (DMR). By this process, DNA methylation patterns acquired during gametogenesis that are unnecessary for zygotic development are erased. The DMR patterns of various mammalian species have been studied; however, they do not seem to have a conserved pattern. We examined early goat embryos to find conforming rules underlying mammalian DMR patterns. Immunocytochemical results showed that the overall level of DNA methylation was not greatly changed during the pronucleus stage. At the two-cell stage, active demethylation occurred and simultaneously affected both parental DNAs, resulting in a global loss of 5methylcytosine. The level of DNA methylation was lowest in the four-cell stage, with increased de novo methylation during the eight-cell stage. Histone $\mathrm{H} 3$-lysine 9 was gradually trimethylated in the sperm-derived chromatin, continuing from the pronucleus stage through the two-cell stage. This goat DMR pattern is novel and distinct from the DMRs of other mammalian species. The more mammalian species we included for DMR analysis, the more multifarious patterns we obtained, adding an extra diversity each time to the known mammalian DMR patterns. Nevertheless, the evolutionary significance and developmental consequence of such diverse DMR patterns are currently unknown.
\end{abstract}

KEY WORDS: DMR, reprogramming, histone methylation, preimplantation development, epigenetics

DNA methylation patterns are relatively stable and heritable, and involved in some specialized biochemical processes, such as allele-specific gene expression (Reik and Walter, 2001), X chromosome inactivation (Heard, 2004), and heritable gene silencing of parasitic sequence elements (Yoder et al., 1997). Notwithstanding the stable and heritable features, DNA methylation patterns go through a reset process at certain thresholds of development, which is necessary to prepare the genome for subsequent development. The conceptual significance of DNA methylation reprogramming (DMR) lies in the removal of the differentiation-associated DNA methylation and re-writing of the epigenetic draft to attain nuclear pluripotency (Kang et al., 2003; Li, 2002; Reik et al., 2001; Rideout et al., 2001). The preimplantation stage is one stage in which DMR (Park et al., 2007) is necessary.

Pre-implantation stage DMR patterns have been characterized for several mammalian species. The first DMR pattern to be characterized was that of the mouse. In mice, DMR consists of active DNA demethylation in the male pronucleus (Mayer et al., 2000; Oswald et al., 2000; Santos et al., 2002) and a passive mode of demethylation that mainly affects the female DNA during the cleavage stages (Dean et al., 2001; Mayer et al., 2000; Monk et al., 1987; Oswald et al., 2000; Razin and Shemer, 1995; Rougier et al., 1998; Sanford et al., 1984). When the mouse DMR pattern was first reported, the majority in the field assumed it to be canonical and tacitly took it for granted that other mammalian species might have similar, if not the same, DMR patterns (Kang et al., 2003). Analyses of other mammalian species later, however, have revealed varied DMR patterns. No active demethylation has been detected in sheep (Beaujean et al., 2004a; Beaujean et al., 2004b), rabbits (Beaujean et al., 2004a; Shi et al., 2004), or

\footnotetext{
Abbreviations used in this paper: 5-azadC, 5-azadeoxycytidine; 5-MeC, 5methylcytosine; DMR, DNA methylation reprogramming; fPN, female pronucleus; H3K9me3, H3-lysine 9 trimethylation; Pb, polar body.
}

\footnotetext{
*Address correspondence to: Sang-Tae Shin. College of Veterinary Medicine, Chungnam National University, Daejon, 305-764, South Korea.

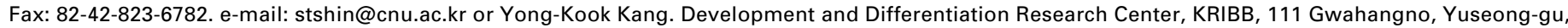
Daejeon 305-806, South Korea. Fax: +82-42-860-4608. e-mail: ykkang@kribb.re.kr
} 
pigs (Jeong et al., 2007a; Jeong et al., 2007b). The lack of active demethylation events in these species suggests that the murine DMR pattern should be taken as unique rather than standard. Even more dynamic DMR patterns than that of the mouse has been observed; the bovine DMR pattern includes successive active DNA demethylation and de novo DNA methylation during pronucleus development (Park et al., 2007). Taken together, it appears that individual species have their own patterns of DMR. The distinct DMR strategies used by different mammalian species are likely to produce different gene expression profiles, at least temporarily, and may influence the complicated processes of early embryonic development and differentiation.

We examined goat embryos at the pre-implantation stage in an attempt to determine uniform rules underlying mammalian DMR patterns. We found that, at the two-cell stage, an active mode of demethylation affects both parental DNA simultaneously, resulting in a global loss of 5-methylcytosine (5-MeC) in the two-cell nuclei. The DNA methylation level was lowest at the four-cell stage and increased during the eight-cell stage. This DMR pattern

A
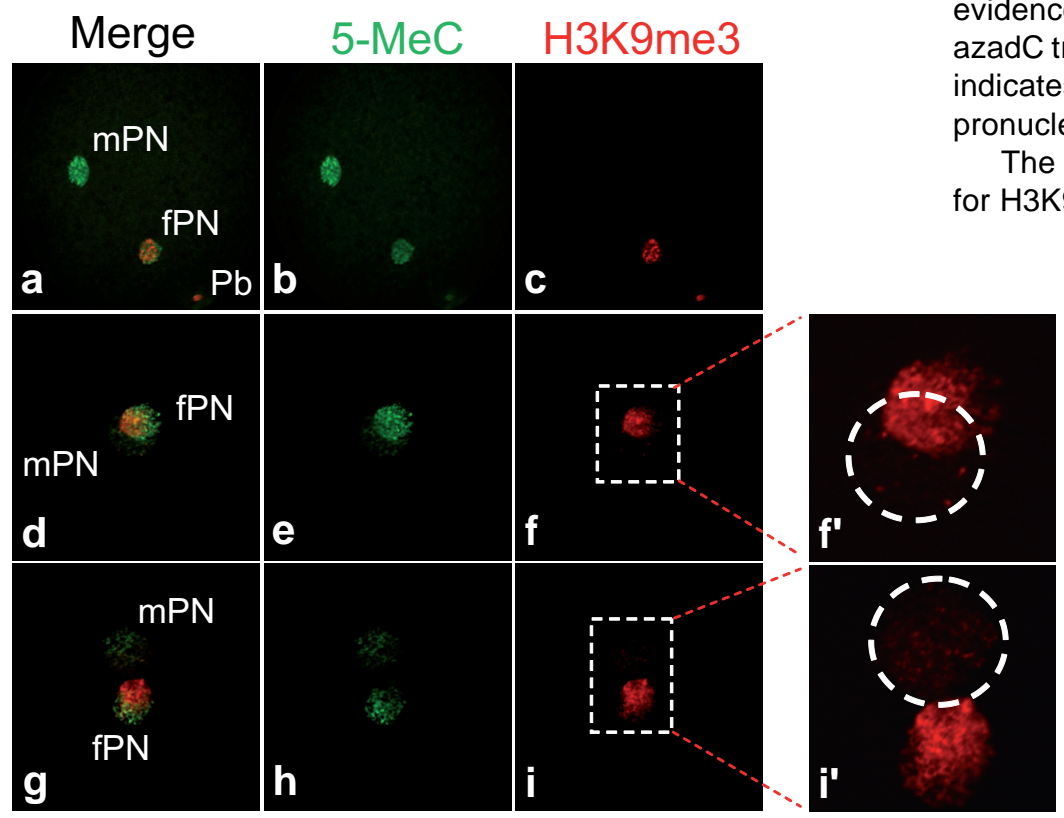

B

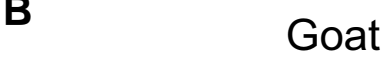

Goat
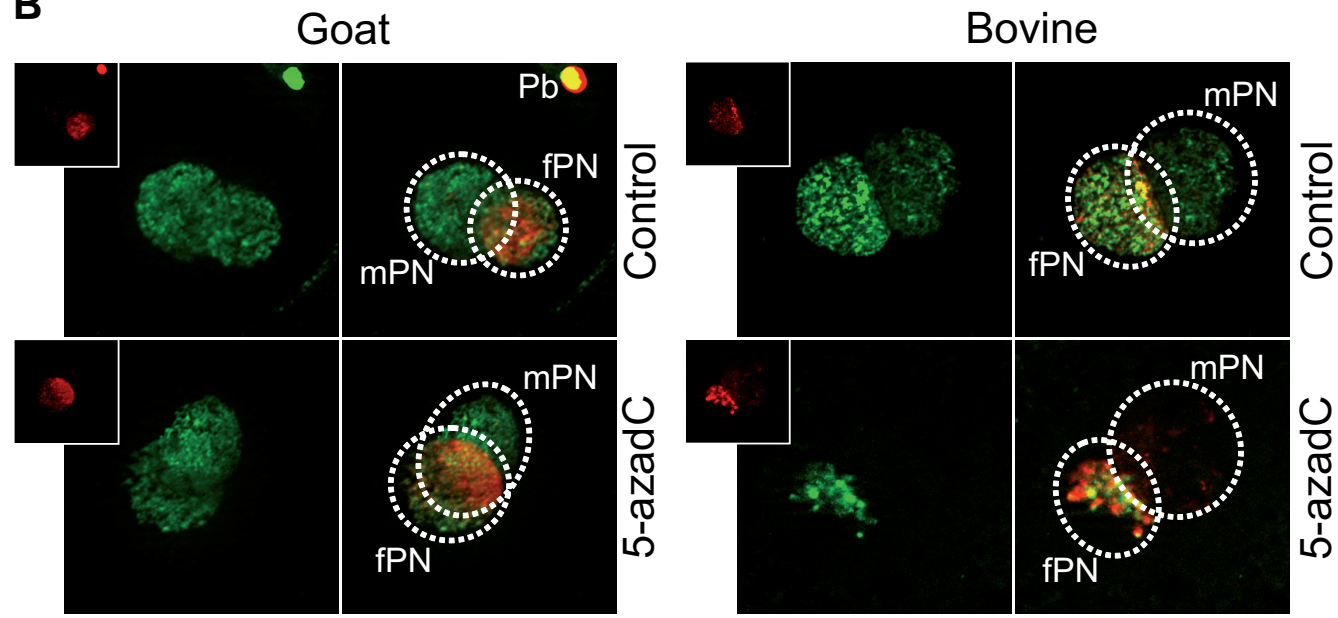

\section{Results}

is novel and unique to goat embryos in that the active DNA demethylation occurs in both male and female DNA at a single cleavage stage.

\section{DNA and H3K9 methylation states in goat zygotes derived in vivo}

We immunocytochemically examined the global states of DNA methylation and trimethylation at histone $\mathrm{H} 3$-lysine 9 (H3K9me3) during early goat development. We found that goat zygotes at different time points of development possessed sufficient 5methylcytosine (5-MeC) signals in them (Fig. 1A), with the level of the male pronucleus equivalent to, or slightly lower than, that of the female one at later pronucleus stage (Fig. $1 \mathrm{Ae}$ and $\mathrm{h}$ ). That the obvious signals for $5-\mathrm{MeC}$ were present in the male pronucleus implied that DNA demethylation did not occur thoroughly in the zygote stage. When we treated goat zygotes with 5-azadeoxycytidine (5-azadC), we observed no sign of a global loss of DNA methylation in the male goat pronucleus (Fig. 1B); different from the goat zygotes, bovine zygotes as a control revealed evidence of active demethylation in the male pronucleus by the 5azadC treatment, as shown before (Park et al., 2007). This result indicates the lack of an active DNA demethylation event in the pronucleus-stage goat embryos.

The female pronucleus was shown to harbor intense signals for H3K9me3 (Fig. 1Ac-i). In contrast, the male pronucleus had either no (Fig. 1Ac) or weak H3K9me3 signals (Fig. 1 Af-f' and $i-i$ '). These results indicate that the paternal H3K9me3 signals, which are initially absent in

Fig. 1. DNA methylation and histone H3-lysine 9 trimethylation ( $\mathrm{H} 3 \mathrm{~K} 9 \mathrm{me} 3$ ) in goat zygote pronuclei. In vivo-derived goat zygotes were fixed before immunostaining using anti-5-methylcytosine (5-MeC) antibody (green) and anti-H3K9me3 antibody (red) (12 hours apart, $n=18$ and $n=20$ ). The goat male pronucleus is morphologically distinct from the female version and is identified as follows: 1) usually slightly larger than the female counterpart and distal from the polar body; 2) relatively decondensed; and 3) weakly stained for H3K9me3 compared with the female pronucleus (Park et al., 2007). The female pronucleus (fPN) had stronger $\mathrm{H} 3 \mathrm{~K} 9 \mathrm{me} 3$ signals than the male pronucleus (MPN). The boxed area in panels $(f, i)$ in (A) are enlarged in panels $\left(f^{\prime}, i^{\prime}\right)$, respectively. (B) 5-azadeoxycytidine (5-azadC) treatment. In vivo-derived goat zygotes were cultured in the absence (Control) or presence (5-azadC) of 5 $\mu M$ 5-azadC for 12 hours and then fixed for immunostaining. 5-azadC treatment made no difference in the methylation levels of the male goat pronucleus. As a control, bovine zygotes were also included; 5-azadCtreated bovine zygotes contain no 5$\mathrm{MeC}$ signal in the male pronuclei. Insets show H3K9me3 images. Nuclear boundary is indicated as a dashed circle. Pb, polar body. 


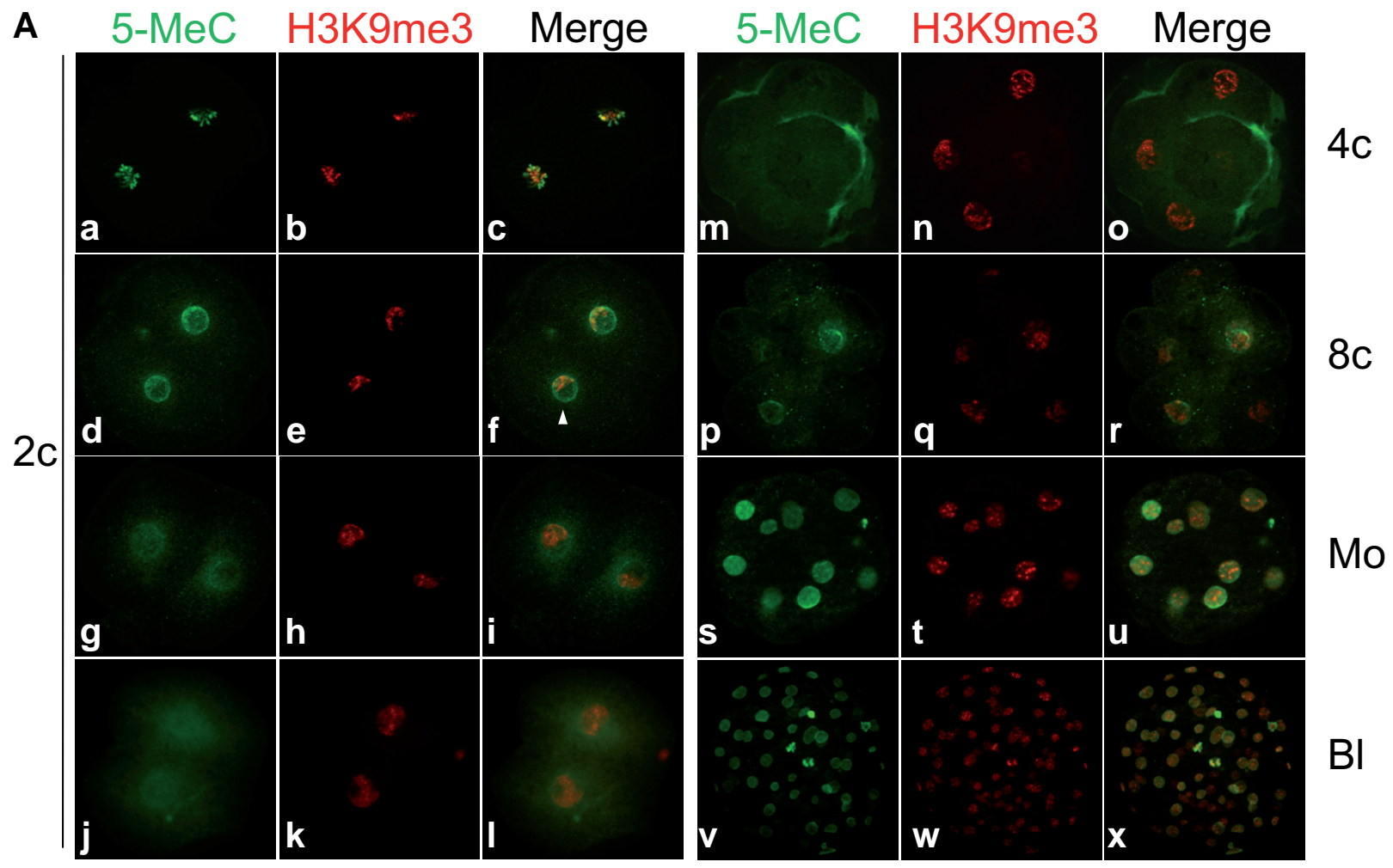

B

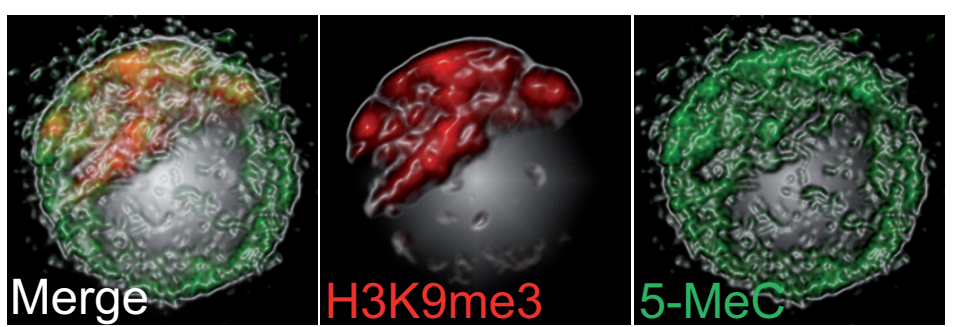

Fig. 2. Features of DNA and H3K9 methylation in cleavage-stage goat embryos. (A) Changes in the DNA methylation and H3K9me3 states. Cleavagestage goat embryos derived from in vivo fertilization were doubly stained for $5-\mathrm{MeC}$ and H3K9me3. Twocell stage embryos (2c) were arranged by the extent of H3K9me3 and 5-MeC in the nucleus (b-k). 4c, four-cell

embryo; 8c, eight-cell embryo; Mo, morula; Bl, blastocyst. (B) Nuclear compartmentalization of a two-cell nucleus. The two-cell embryonic nucleus shown in panel (f) (arrowhead) was retouched using Photoshop software (version 7) and a plastic wrap filter.

spermic genomic materials, develop gradually after the protamine-histone exchange of chromatin remodeling (McLay and Clarke, 2003). The amount of paternal H3K9me3 was timedependent, relying on how far the male pronucleus had advanced through the cell cycle. However, the overall levels of paternal $\mathrm{H} 3 \mathrm{~K} 9 \mathrm{me} 3$ in the goat zygotes were much lower than the maternal level.

\section{Occurrence of active DNA demethylation and H3K9 methyla- tion in two-cell stage goat embryos}

The gradually developing $\mathrm{H} 3 \mathrm{~K} 9 \mathrm{me} 3$ signals in the paternal pronucleus did not reach a level equivalent to the maternal pronucleus. Immunostaining revealed that the nucleus of two-cell stage goat embryos were compartmentalized by H3K9me3 signals, with half the nucleus being methylated and the other half being non-methylated (Fig. 2Ae-h and Fig. 2B). The same nucleus had a relatively even spread of $5-\mathrm{MeC}$ signal to the whole nucleoplasm compared to the H3K9me3 signal. The vacant half of the nucleus, which was assumed to be occupied by the chromatins of male origin, was gradually filled up with $\mathrm{H} 3 \mathrm{~K} 9 \mathrm{me} 3$ signals as the two-cell embryos advanced (Fig. 2Ak). From the four-cell stage onwards, the level of $\mathrm{H} 3 \mathrm{~K} 9 \mathrm{me} 3$ was mostly maintained without significant change (Fig. 2An-w).

As the H3K9me3 signals progressively increased, the 5-MeC levels adjusted in the opposite direction. We noticed that the nuclei of two-cell embryos had a broad spectrum of DNA methylation levels, ranging from distinct (Fig. 2Aa-d) to vague (Fig. 2Agj). When two-cell embryos were sorted by the extent of H3K9 trimethylation, which is associated with cell cycle progression, we found that the vivid 5-MeC signals in earlier two cell-stage embryos became fainter at later stages (Fig. 2Aa-j), leaving only trace signals of DNA methylation in the nucleus. Such a change in DNA methylation was ascertained when two-cell embryos were placed side by side with one-cell zygotes; the pronuclei of the onecell goat zygote had 5-MeC signals, but the two-cell nuclei did not (Fig. 3A).

\section{De novo DNA methylation occurs in eight-cell stage goat embryos}

The global loss of 5-MeC during the two-cell stage resulted in the lowest DNA methylation state in the four cell-stage embryos (Fig. 2Am), followed by de novoDNA methylation during the eight- 
cell stages as 5-MeC signals began to appear in some of the embryonic nuclei (Fig. 2Ap). Figure 3B clearly shows an increase in the 5-MeC level during the transition from the four- to eight-cell stage. DNA methylation signals were eventually present in all embryonic nuclei when the goat embryos reached the morula stage (Fig. 2As). Blastocysts, which consist of two different cell lineages with different developmental potential, such as the innercell mass (ICM) and trophectoderm (TE), showed no regional differences in the level of DNA methylation (Fig. 2Av). This pattern of DNA methylation reprogramming differed from those of pig and mouse; the former showed no change in DNA methylation level during the cleavage stages and the latter displayed a progressive demethylation (Jeong et al., 2007a), so none of them resembled the reprogramming pattern of goat embryos. In summary, the global level of 5-MeC was not greatly changed during the pronucleus stage. At the two-cell stage, an active mode of demethylation occurred and both parental DNAs were concurrently affected, resulting in a genome-wide loss of $5-\mathrm{MeC}$. The level of 5-MeC was lowest at the four-cell stage and increased again during the eight-cell stage via de novo DNA methylation. H3K9me3 gradually developed in the sperm-derived chromatin, continuing from the pronucleus stage through the two-cell stage.

\section{Discussion}

We recently showed that different mammalian embryos have distinct patterns of DMR at the zygote stage (Park et al., 2007). Type I species, including rodents, undergo active DNA demethylation without global H3K9me3 in the male pronucleus. Type II species maintain the paternal DNA methylation level, and acquired substantial level of $\mathrm{H} 3 \mathrm{~K} 9 \mathrm{me} 3$, as recently shown in sheep (Beaujean et al., 2004a) and pig zygotes (Jeong et al., 2007a; Jeong et al., 2007b). Bovine zygotes belong to type III species
(Park et al., 2007); the sperm-derived pronucleus looked as if it is partially demethylated. Goat zygotes, looking only at the outside, bear a resemblance to bovine zygotes in that they exhibit fractional demethylation in some of the male pronucleus (Fig. 1e). In spite of the outward similarity of the bovine and goat zygotes, the internal circumstances are quite different; there are completely different DMR processes advancing inward. Bovine zygotes have active demethylation and ensuing re-methylation in the male pronucleus, whereas the goat zygote does not have such a drastic change in the DNA methylation. As shown in Fig. 2A, both active DNA demethylation and re-methylation take place in goat embryos during the cleavage stages. This observation suggests that goat embryos should be re-categorized as a new DMR group of TypeIV.

Zygote-stage mammalian eggs have bundled parental genomes sequestered apart from one another in the pronuclei. The spatial separation between the male and female pronuclei is thought to guarantee global demethylation and intensively affect the male DNA. Thus, the pronucleus stage is assumed to be the most dynamic and relevant period during which the differential methylation and demethylation of the parental DNA take place. In line with this, active DNA demethylation events have been detected in the pronucleus of sperm origin in the mouse, rat, and bovine; therefore, the female genome (and its pan-chromosomal epigenetic states) appears somehow 'protected' as a pronuclear membrane-bound entity from the process of seemingly disastrous global DNA demethylation. However, in this study, we observed full-scale DNA demethylation in the two-cell nuclei (Fig. 2A). Notably, both the female and male genomes were concurrently demethylated within the same nucleus, without the former being spatially isolated from the latter. There are some possible explanations for this observation. Goat embryos may have an escape strategy. Both parental sets of genomes, even if they share the
A

Fig. 3. Changes in the DNA methylation state at different goat embryo cleavage stages. (A-B) Individual photos were taken of different cleavage-stage embryos to easily compare relative DNA methylation levels between the zygote and two-cell embryo $(A, 1 \mathrm{C}$ vs. 2c) or four-cell and eight-cell embryos (B, $4 c$ vs. $8 C$ ). The pronuclei (A) and eight-cell nuclei (B) clearly have 5-MeC signals (white arrows), but not the two-cell (A) or four-cell nuclei (B) lyellow arrowheads). As a control, H3K9me3 signals appear in at all stages. (C) Changes in the levels of DNA methylation and H3K9me3 in preimplantation stage goat embryos. The most dynamic change was seen during the two-cell stage;

the 5-MeC (green) and H3K9me3 (red) levels changed in opposite directions. De novo DNA methylation occurred after the genome-wide establishment of H3K9me3. As references, the changing patterns of DNA and H3K9me3 methylation in early mouse (Jeong et al., 2007a; Santos et al., 2002) and pig (Jeong et al., 2007a) embryos are also illustrated below.

B

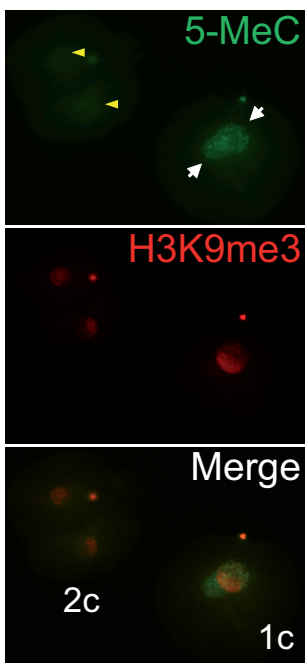

1c vs. 2c

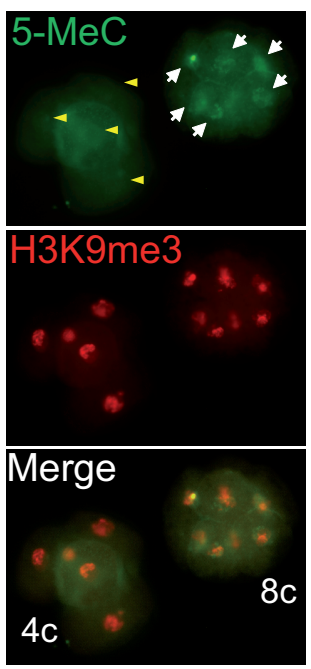

4c vs. 8c

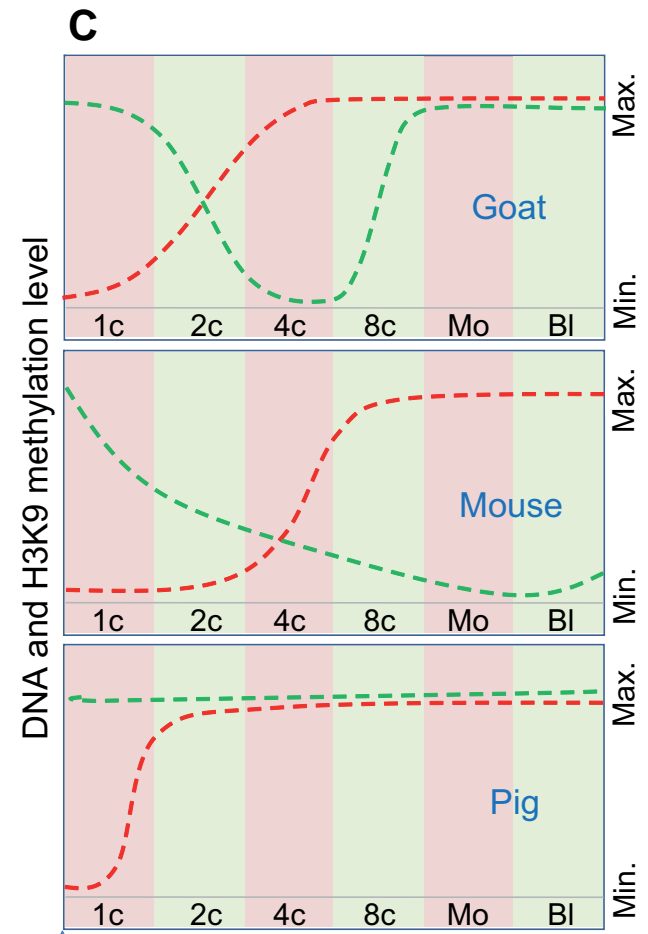

Fertilization 
same nucleoplasmic space, are not necessarily affected by active demethylation processes. The female genome has already been filtered through the pronucleus stage, suggesting that it has sufficient time to secure itself against the upcoming genome-wide demethylation event. On the other hand, the male genome acquires specific indications through the pronucleus stage that are preferentially perceived by the machinery of active DNA demethylation. Alternatively, at a regional level, epigenetically important genomic regions, such as imprinted loci, may possess innate safety devices that ensure the local epigenetic states are protected from destructive modification. Provided that there are such control mechanisms of local DNA methylation states and tactics to evade detrimental epimutations, a global demethylation process occurring simultaneously over the female and male DNA would not necessarily be harmful; instead, simultaneous demethylation would modify both parental DNAs through the same mechanisms using common machinery, so the resulting diploid genome would be regulated in an efficient and coordinate manner.

The timing of $\mathrm{H} 3 \mathrm{~K} 9 \mathrm{me} 3$ in the spermic chromatin of the goat embryo is also distinct from that of other species. Bovine and porcine sperm-derived chromatins attain sufficient H3K9me3 signals during the pronucleus stage (Jeong et al., 2007b; Park et al., 2007). In mice, the male chromatin does not acquire H3K9me3 signals until the four-cell stage when the embryonic nuclei become enriched with H3K9me3 signals (Liu et al., 2004; Yeo et al., 2005). Regarding the establishment of an $\mathrm{H} 3 \mathrm{~K} 9 m e 3$ pattern, the goat is not equal to these species. For the sperm-derived chromatin in goat embryos, H3K9me3 began in the zygote stage similar to the bovine and porcine species, but the level of methylation remained low and never increased to the level of the female chromatin (Fig. $2 A f^{\prime}$ and i'). The methylation state was maintained until the early two-cell stage, and the unbalanced H3K9 trimethylation of the parental genomes produced a compartmentalized nucleus in regards to the H3K9me3 signal (Fig. 2B), as previously seen in twocell mouse embryos (Yeo et al., 2005). As the embryos advanced to a later two-cell stage, the male $\mathrm{H} 3 \mathrm{~K} 9 \mathrm{me} 3$ gradually increased to a level comparable to that of female H3K9me3 (Fig. 2Ak). Summarizing the immunostaining results from various mammalian embryos to date, the sperm-derived chromatin might be grossly modified by $\mathrm{H} 3 \mathrm{~K} 9 \mathrm{me} 3$ during the four-cell stage at the latest. We previously proposed that de novo $\mathrm{H} 3 \mathrm{~K} 9$ methylation directs de novoDNA methylation (Park et al., 2007); the results of this study with goat embryos also conform to the proposition that the development of H3K9me3 at the two-cell stage precedes de novo DNA methylation at the eight-cell stage (Fig. 3C).

In conclusion, goat embryos exhibit a DMR pattern distinct from the known patterns of other mammalian species; both male and female DNA are concurrently demethylated during the two-cell stage with subsequent de novo methylation, mainly during the eight-cell stage. The results agree with previous findings that different mammalian species have their own DMR patterns. The evolutionary significance of the diverse DMR patterns and the developmental consequence of species-specific reprogramming processes are yet to be studied.

\section{Materials and Methods}

\section{Antibodies}

A monoclonal anti-5-MeC antibody was purchased from Eurogentech (MMS-900S-B). Antibody that specifically recognizes $\mathrm{H} 3 \mathrm{~K} 9 \mathrm{me} 3$ was pur- chased from Upstate biotechnologies. Chicken anti-mouse or anti-rabbit IgG secondary antibodies (Alexa- Fluor 488/594-conjugated form, Molecular Probes) were used to detect the individual primary antibodies.

\section{Collection of goat embryos}

Unless otherwise mentioned, all chemicals used in this study were purchased form Sigma Chemical Co. Goat fertilized oocytes were collected from superovulated females 2 to 3 -year-old pluriparous Korean native goats (Lee et al., 2000). The estrous cycle of female goats was synchronized by ear implantation of $6 \mathrm{mg}$ norgestomet implants (Synchromate-B, Rhone Merieux, Inc., Athens, GA) for 14 days. Superovulation was induced by a combined injection of FSH and hCG. A total of $5.6 \mathrm{mg}$ of $\mathrm{FSH}$ (Ovagen, Immuno-Chemical Products, New Zealand) was given to goats over a 4-day period as injection, twice daily, starting 2.5 days before implant removal and continuing to 1 day after removal. A single injection of hCG (100 IU) was given at the time of last FSH injection for the induction of ovulation. Within $24 \mathrm{~h}$ following implant removal, the female goats were mated with male goat. Sixty-eight hrs following implant removal, Oviducts of female goats were surgically recovered and flushed in retrograde fashion with sterile PBS as previously reported (Lee et al., 1997).

\section{5-azadeoxycytidine treatment}

In vivo-derived goat zygotes were cultured in the presence of $5 \mu \mathrm{M} 5$ azadC (Sigma Aldrich) for 12 hours and then fixed for immunostaining. Procedure for the treatment of bovine zygotes with 5-azadC was described elsewhere (Park et al., 2007). Briefly, the oocytes-spermatozoa complexes were transferred to IVF medium containing 5 uM 5-azadC eight hours after the commencement of in vitro fertilization, and cultured for another 12 hours. The samples were fixed for immunostaining.

\section{Immunostaining early embryos}

Immunostaining procedure appeared elsewhere (Jeong et al., 2007b). Briefly, embryos were fixed in 4\% formaldehyde in PBS (Gibco) for 30 minutes at $4{ }^{\circ} \mathrm{C}$, followed by three times of 20 -minute wash in $0.02 \%$ Tween 20(Fisher Scientific) in PBS and permeabilized by $0.25 \%$ Triton X100 (MP Biomedicals) in PBS for one hours at room temperature. The embryos were treated with $4 \mathrm{~N} \mathrm{HCl}$ (Sigma) for 30 minutes at room temperature, neutralized with $0.1 \mathrm{M}$ Tris $-\mathrm{HCl}(\mathrm{pH} 8.0)$ and blocked for one hour at room temperature in 2\% BSA (Sigma), 0.02\% Tween 20 in PBS. Primary antibody incubations (diluted by $1: 100-300$ ) were carried out in the blocking solution for one hour at $37^{\circ} \mathrm{C}$, followed by several washes in $0.02 \%$ Tween 20 in PBS. Secondary antibodies (diluted to 1:300-500) were incubated for 30 minutes at room temperature, followed by several washes. Embryos were mounted on Poly-prep slides (Sigma) and, after dry at room temperature, observed with Karl Zeiss Axiovert 200M fluorescence microscope equipped with ApoTome. Most staining experiments were repeated at least three times each with 20 or more embryos. Images were captured digitally using different filter sets and merged using Axiovision (v4.5) or Adobe Photoshop software (v7.0).

\section{Acknowledgement}

This work was supported by grants from TDPAF (ARPC) and KRIBB Research Initiative Program (KRIBB).

\section{References}

BEAUJEAN, N., HARTSHORNE, G., CAVILLA, J., TAYLOR, J., GARDNER, J., WILMUT, I., MEEHAN, R., AND YOUNG, L. (2004a). Non-conservation of mammalian preimplantation methylation dynamics. Curr Bio/14: R266-267.

BEAUJEAN, N., TAYLOR, J.E., MCGARRY, M., GARDNER, J.O., WILMUT, I., LOI, P., PTAK, G., GALLI, C., LAZZARI, G., BIRD, A., ET AL. (2004b). The effect of interspecific oocytes on demethylation of sperm DNA. Proc Nat/ Acad Sci USA 101: $7636-7640$

DEAN, W., SANTOS, F., STOJKOVIC, M., ZAKHARTCHENKO, V., WALTER, J., 
WOLF, E., AND REIK, W. (2001). Conservation of methylation reprogramming in mammalian development: aberrant reprogramming in cloned embryos. Proc Natl Acad Sci USA 98: 13734-13738.

HEARD, E. (2004). Recent advances in X-chromosome inactivation. Curr Opin Cell Biol 16: 247-255.

JEONG, Y.S., YEO, S., PARK, J.S., KOO, D.B., CHANG, W.K., LEE, K.K., AND KANG, Y.K. (2007a). DNA methylation state is preserved in the sperm-derived pronucleus of the pig zygote. Int J Dev Bio/51: 707-714.

JEONG, Y.S., YEO, S., PARK, J.S., LEE, K.K., AND KANG, Y.K. (2007b). Gradual development of a genome-wide H3-K9 trimethylation pattern in paternally derived pig pronucleus. Dev Dyn 236: 1509-1516.

KANG, Y.K., LEE, K.K., AND HAN, Y.M. (2003). Reprogramming DNA methylation in the preimplantation stage: peeping with Dolly's eyes. Curr Opin Cel/ Biol15: 290-295.

LEE, C.S., FANG, N.Z., KOO, D.B., LEE, Y.S., ZHENG, G.D., OH, K.B., YOUN, W.S., HAN, Y.M., KIM, S.J., LIM, J.H., ET AL. (2000). Embryo recovery and transfer for the production of transgenic goats from Korean native strain, Capra hircus aegagrus. Small Rumin Res 37: 57-63.

LEE, W.K., HAN, Y.M., SHIN, S.T., LEE, D.H., YOO, O.J., AND LEE, K.K. (1997). In vitro development of DNA-injected embryos co-cultured with goat oviduct epithelial cells in Korean native goats (Capra hircus aegagrus). Theriogenology 47: 1115-1123.

LI, E. (2002). Chromatin modification and epigenetic reprogramming in mammalian development. Nat Rev Genet 3: 662-673.

LIU, H., KIM, J.M., AND AOKI, F. (2004). Regulation of histone H3 lysine 9 methylation in oocytes and early pre-implantation embryos. Development 131 : 2269-2280.

MAYER, W., NiVELEAU, A., WALteR, J., FUNDELE, R., AND HAAF, T. (2000). Demethylation of the zygotic paternal genome. Nature 403: 501-502.

MCLAY, D.W., AND CLARKE, H.J. (2003). Remodelling the paternal chromatin at fertilization in mammals. Reproduction 125: 625-633.

MONK, M., BOUBELIK, M., AND LEHNERT, S. (1987). Temporal and regional changes in DNA methylation in the embryonic, extraembryonic and germ cell lineages during mouse embryo development. Development 99: 371-382.
OSWALD, J., ENGEMANN, S., LANE, N., MAYER, W., OLEK, A., FUNDELE, R., DEAN, W., REIK, W., AND WALTER, J. (2000). Active demethylation of the paternal genome in the mouse zygote. Curr Biol10: 475-478.

PARK, J.S., JEONG, Y.S., SHIN, S.T., LEE, K.K., AND KANG, Y.K. (2007). Dynamic DNA methylation reprogramming: active demethylation and immediate remethylation in the male pronucleus of bovine zygotes. Dev Dyn236:25232533.

RAZIN, A., AND SHEMER, R. (1995). DNA methylation in early development. Hum Mol Genet 4: 1751-1755.

REIK, W., DEAN, W., AND WALTER, J. (2001). Epigenetic reprogramming in mammalian development. Science 293: 1089-1093.

REIK, W., AND WALTER, J. (2001). Genomic imprinting: parental influence on the genome. Nat Rev Genet 2: 21-32.

RIDEOUT, W.M., 3RD, EGGAN, K., AND JAENISCH, R. (2001). Nuclear cloning and epigenetic reprogramming of the genome. Science 293: 1093-1098.

ROUGIER, N., BOURC'HIS, D., GOMES, D.M., NIVELEAU, A., PLACHOT, M., PALDI, A., AND VIEGAS-PEQUIGNOT, E. (1998). Chromosome methylation patterns during mammalian preimplantation development. Genes Dev 12: 2108-2113.

SANFORD, J., FORRESTER, L., CHAPMAN, V., CHANDLEY, A., AND HASTIE, N. (1984). Methylation patterns of repetitive DNA sequences in germ cells of Mus musculus. Nucleic Acids Res 12: 2823-2836.

SANTOS, F., HENDRICH, B., REIK, W., AND DEAN, W. (2002). Dynamic reprogramming of DNA methylation in the early mouse embryo. Dev Bio/241: 172182.

SHI, W., DIRIM, F., WOLF, E., ZAKHARTCHENKO, V., AND HAAF, T. (2004). Methylation reprogramming and chromosomal aneuploidy in in vivo fertilized and cloned rabbit preimplantation embryos. Biol Reprod 71: 340-347.

YEO, S., LEE, K.K., HAN, Y.M., AND KANG, Y.K. (2005). Methylation changes of lysine 9 of histone $\mathrm{H} 3$ during preimplantation mouse development. Mo/Cel/s20: 423-428.

YODER, J.A., WALSH, C.P., AND BESTOR, T.H. (1997). Cytosine methylation and the ecology of intragenomic parasites. Trends Genet 13: 335-340. 


\section{Further Related Reading, published previously in the Int. J. Dev. Biol.}

See Special Issue Pattern Formation edited by Michael K. Richardson and Cheng-Ming Chuong at: http://www.ijdb.ehu.es/web/contents.php?vol=53\&issue=5-6

Gene expression in the placenta: maternal stress and epigenetic responses Ciprian P. Gheorghe, Ravi Goyal, Ashwani Mittal and Lawrence D. Longo Int. J. Dev. Biol. in press (doi: 10.1387/ijdb.082770cg)

Mammalian hibernation: differential gene expression and novel application of epigenetic controls Pier Morin Jr. and Kenneth B. Storey Int. J. Dev. Biol. (2009) 53: 433-442

Interplay between DNA methylation, histone modification and chromatin remodeling in stem cells and during development Kohta Ikegami, Jun Ohgane, Satoshi Tanaka, Shintaro Yagi, and Kunio Shiota Int. J. Dev. Biol. (2009) 53: 203-214

Epigenetic asymmetry in the zygote and mammalian development Robert Feil

Int. J. Dev. Biol. (2009) 53: 191-201

Hypomethylation of paternal DNA in the late mouse zygote is not essential for development Zbigniew Polanski, Nami Motosugi, Chizuko Tsurumi, Takashi Hiiragi and Steffen Hoffmann Int. J. Dev. Biol. (2008) 52: 295-298
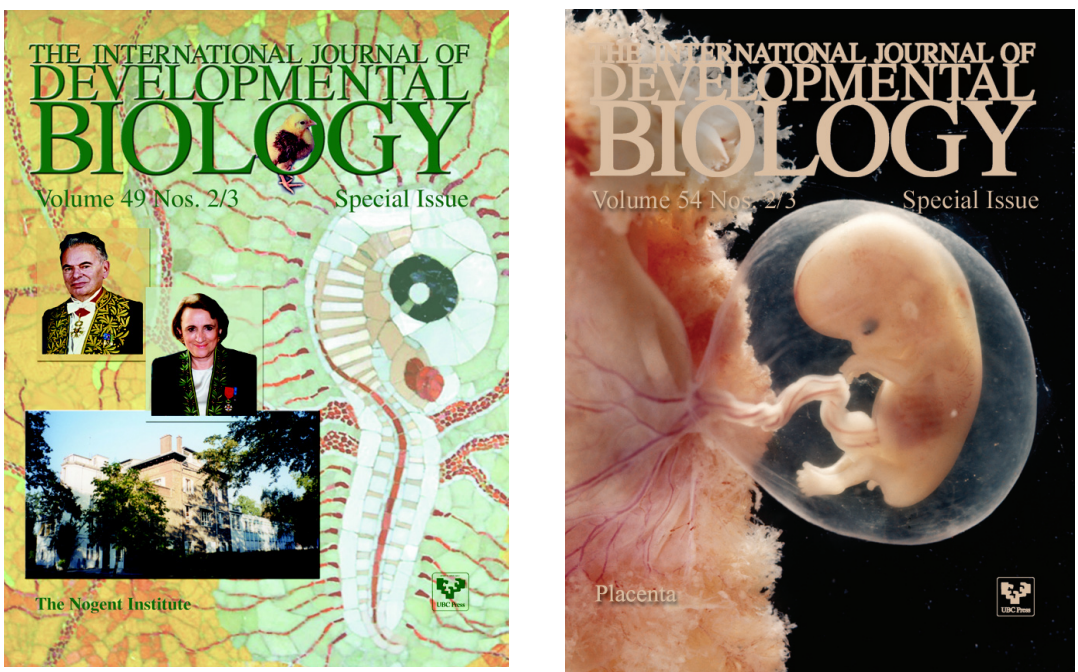

5 yr ISI Impact Factor $(2009)=3.253$
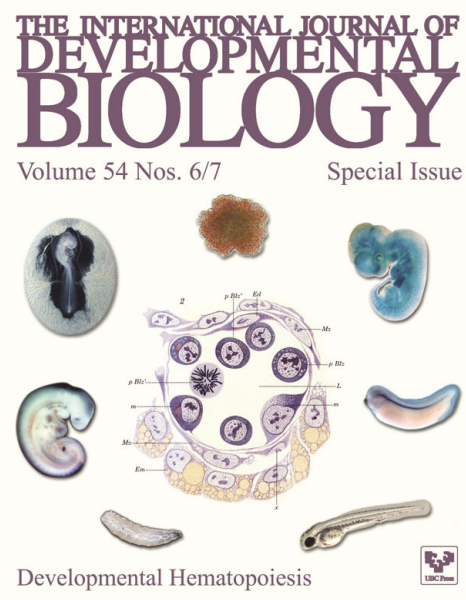